\title{
Haemodialysis services in the northeastern region of Iran
}

\author{
Behzad Kiani, ${ }^{1}$ Nasser Bagheri, ${ }^{2}$ Ahmad Tara, ${ }^{3}$ Benyamin Hoseini, ${ }^{1}$ Mahmood Tara ${ }^{1}$ \\ ${ }^{1}$ Department of Biomedical Informatics, Faculty of Medicine, Mashhad University of Medical Sciences, \\ Mashhad, Iran; ${ }^{2}$ Department of Health Services Research and Policy, College of Medicine, Biology and \\ Environment, Australian National University, Canberra, Australia; ${ }^{3}$ Department of Nephrology, Shahid \\ Modarres Hospital, Shahid Beheshti University of Medical Sciences, Tehran, Iran
}

\begin{abstract}
Chronic kidney disease is growing and the current estimated global prevalence exceeds $13 \%$. As the use of haemodialysis machines for patients with end stage renal disease increases survival considerably, it is critical to plan correctly for the allocation of these machines. This study aimed to develop a geographical information systems (GIS)-based approach to predict the need for this service in the northeastern region of Iran taking into account where patients live and where haemodialysis is the most needed and identifying areas with poor access to haemodialysis centres. Patients were interviewed to obtain self-reported actual travel time and the inverse distance-weighting algorithm was used to determine access in each area. The prediction is based on the domestic
\end{abstract}

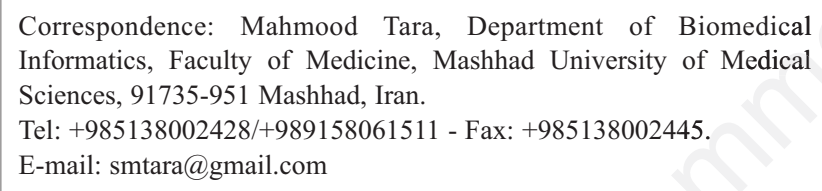

Key words: Actual accessibility; Geographic information systems; Haemodialysis machine; Interpolation; Iran.

Acknowledgments: the authors would like to make special thanks to Dr. Mohsen Shahriari for his contribution to data gathering.

Contributions: $\mathrm{BK}, \mathrm{BH}$, data collecting; $\mathrm{BK}$, analysing and manuscript writing; MT, AT, NB, manuscript reviewing; BK, AT, MT, study designing.

Conflict of interest: the authors declare no potential conflict of interest.

Funding: the work was supported by a grant [931059] from Mashhad University of Medical Sciences Research Council.

Received for publication: 26 February 2017.

Revision received: 19 April 2017.

Accepted for publication: 20 April 2017.

CCopyright B. Kiani et al., 2017

Licensee PAGEPress, Italy

Geospatial Health 2017; 12:561

doi:10.4081/gh.2017.561

This article is distributed under the terms of the Creative Commons Attribution Noncommercial License (CC BY-NC 4.0) which permits any noncommercial use, distribution, and reproduction in any medium, provided the original author(s) and source are credited. growth rate for haemodialysis services and the estimated active hours of machine use for the next five years. We estimate that six new haemodialysis machines are required in northeastern Iran at the present time with 50 machines required over the next five years. Ashkhane City was identified to have the least access to haemodialysis centres in the study area. Our GIS-based model can be used to investigate not only the need for new haemodialysis machines but also to examine geographic disparities in the allocation of haemodialysis centres and to identify areas most in need of this service. It is important that policymakers consider both spatial and non-spatial dimensions of access to enable better allocation of haemodialysis services ensuring they are targeted to reach those in need.

\section{Introduction}

The prevalence of Chronic kidney disease (CKD) is growing and has reached $13.4 \%$ across the world in 2016 (Hill et al., 2016). The final stage of CKD is end stage renal disease (ESRD) whereby the kidneys do not function well enough to meet the primary needs of the body. These patients need either kidney transplantation or regular dialysis to compensate for the renal performance failure. Dialysis is a procedure for removing waste materials along with maintaining the acid/base balance in the body. In peritoneal dialysis, a liquid is injected into the abdominal cavity followed by its extraction, while haemodialysis requires a machine. The former type of dialysis can be undertaken by patients themselves, either at home or at work after having received training by health professionals, while the latter approach is commonly carried out at a haemodialysis centre due to the high cost of the machines, their maintenance as well as reluctance of patients to use this method at home. Haemodialysis is the most common method of treatment for ESRD patients and continues until a kidney donor available for transplantation is found (Anand et al., 2014). Patients undergoing haemodialysis must visit their centre three times a week (Daugirdas et al., 2015) and there is a proven relationship between these patients' travel time from home to the centre, their mortality rates and health status (Moist et al., 2008). In order to decrease travel time and enhance the quality of the service provided, the allocation of haemodialysis machines should preferably be based on the actual need in the areas where the patients live (Azar, 2009; Rucker et al., 2011; Thompson et al., 2012).

Geographic information systems (GISs) enable the incorporation of spatial as well as non-spatial factors into the process of resource allocation resulting in improved documentation for decisions and interventions. The spatial factors are geography-related, 
e.g., patients' travel time and travel distance to get to the haemodialysis centres, whereas the non-spatial ones are related to haemodialysis services and may refer to the number of available machines, their active hours, the current 12\% growth rate of haemodialysis needs (Omrani-Khoo et al., 2013)) and the number of patients in the district in question. The worldwide recommended maximum time for access to a haemodialysis centre is 30 minutes (Mactier et al., 2007). Thus, available resources should be allocated so that the highest possible number of patients can fit into this time-distance range. The study by Yang et al. (2006) undertaken in USA, as well as the UK Renal Association guidelines published in 2007 , both emphasise that the ratio of registered patients and available haemodialysis machines in each centre should be four at most (Yang et al., 2006; Mactier et al., 2007). The expected durability of a haemodialysis machine has been calculated to be 25,000 hours (Mactier et al., 2007).

The patients' travel time to their haemodialysis centres has been estimated using network analysis in a number of previous studies (Ayyalasomayajula et al., 2011; Matsumoto et al., 2012, 2013; Delmelle et al., 2013; Stephens et al., 2013). Network analysis in GIS is often related to finding solutions to transportation problems such as path-finding and travel time between source and destination (Stentzel et al., 2016). This method obtains the potential travel times to centres. However, there are many factors that can cause a difference between the actual travel time (ATT) and the calculated travel time, e.g., socioeconomic ones (Maheswaran et al., 2003; Diamant et al., 2010), those connected with racial disparities (Saunders et al., 2014) and those connected with transportation (Mao and Nekorchuk, 2013). Therefore, the self-reported ATT is considered a spatial factor in this study, whose aim was to develop a GIS-based approach to predict the number of new haemodialysis machines needed in the northeastern region of Iran in the next five years. An additional aim was to identify areas with poor access to haemodialysis centres.

\section{Materials and Methods}

The study was divided into three phases and carried out using a cross-sectional approach.

\section{Ethical considerations}

All patients were asked to sign a consent form and the study accepted only those agreeing to participate.

\section{Study area and patients}

This study was carried out in North Khorasan Province, Iran, located in the Northeast of the country (Figure 1). The area covers $28,434 \mathrm{~km}^{2}$ and the population was estimated at 811,472 last year

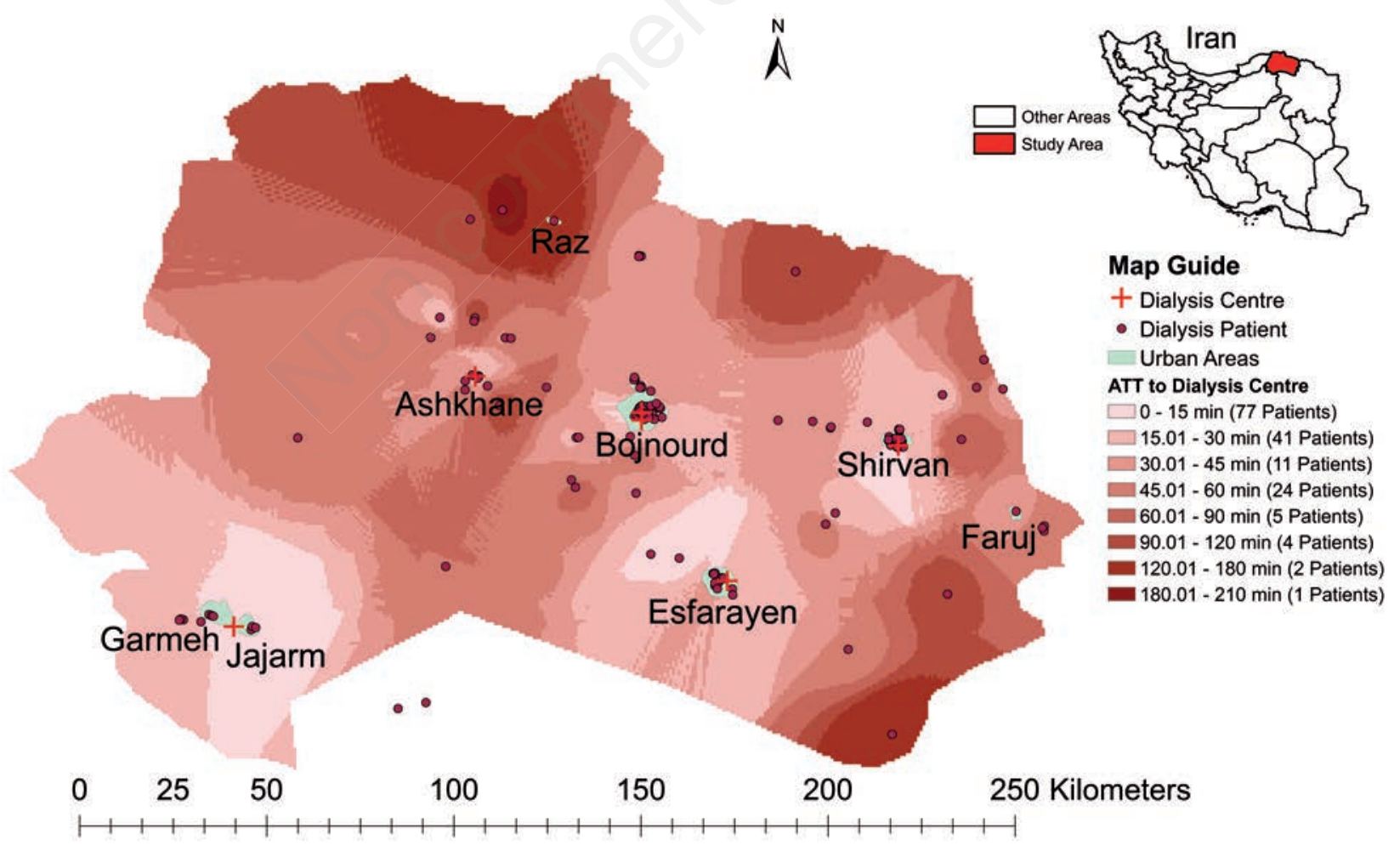

Figure 1. Distribution of patients needing haemodialysis and service centres displayed on an interpolated province map based on actual travel time values. 
(Wikipedia, 2016). The province has six haemodialysis centres located in its six main cities, Ashkhane, Esfaraien, Bojnoord, Jajarm and Shirvan. ESRD patients from all over the province can only be referred for a registered routine service to one of these centres. The province had 203 haemodialysis patients as of December 2015 (this does not include patients visiting from the other provinces), but only 165 of them were available and willing to participate in the study. The distribution of haemodialysis patients' residents and their referred haemodialysis facilities are shown in Figure 1. The points have been jittered for confidentiality reasons. The number of rural patients was 68 and the number of urban ones was 97. Two patients resided outside the provincial borders but were eligible to receive care from the centre in Jajarm.

\section{Phase I: data gathering}

We designed a form and used it to collect information about patients and their residential addresses as well as information regarding each haemodialysis centre, such its address, the number of available haemodialysis machines, previous hours of active machine use and the number of patients receiving service at the centre on a routine basis.

All participants were interviewed and asked about the ATT to their haemodialysis centre without any stop(s) in other places along the way. The response rate was $81 \%$. The ATT results show all responding patients' actual access time to attend their preferred centre (Figure 1).

\section{Phase II: geographic information system analysis}

All patient addresses were geocoded, i.e. assigned geographic coordinates to the street addresses provided. To do this, we developed a programme using C\#.NET, an object-oriented computer language developed by Microsoft as part of the .NET platform to connect to the Google map geocoding Application Programming Interface (API). Our software was able to receive the addresses of the patients and centres and to generate the latitude and longitude for each address. We used a free API license in our software to reduce geocoding costs. If this API was unable to geocode an address, we used an alternative application, Open Street Map (OSM) geocoding API (https://www.openstreetmap.org) in our C\# code. If both methods were unsuccessful, the address was geocod- ed manually. To determine which patients lived more than 30 minutes away from their preferred haemodialysis centres and which ones lived closer than so, the ATTs were interpolated. This means that new data points were constructed within the range of a discrete set of known data points according to Jia et al. (2016), who used Inverse Distance Weighting (IDW), a deterministic algorithm used for multivariate interpolation, to obtain ATTs to health centres at the regional level. A haemodialysis patient layer was created by using the patients' geocoded addresses. The IDW algorithm was run on this layer based on the ATT field value. Figure 2 shows the application of the IDW method estimating the ATT value for a nonsampled patient's address by considering the ATTs of the five nearest patients. The IDW approach gives ATT values closest to the point to be located more influence on the value of the unspecified ATT than those farther away. This calculation was run for all pixels (pixel size $=500$ meters) covering the map of the province. Its output was a raster layer in which each pixel shows the ATT to the haemodialysis centre. A reclassification algorithm based on ATT information was run as well. As a result, two catchments were created for the study area; i) one with less than 30 minutes travel time; and ii) one with more than 30 minutes.

\section{Phase III: development of a geographic information system-based model}

This approach included the development of a model for the prediction of the need for new heamodialysis machines in each area and identification of regions with poor access to haemodialysis centres. The number of active haemodialysis machine hours for the next five years was estimated for each polygon within the $<30$ minutes range of ATT by considering the current number of patients and active machine hours and taking the growth rate of haemodialysis need into account. This was done by dividing the number of patients by the number of haemodialysis machines (machines with $<25,000$ active hours), which should be $\leq 4$ according to (Yang et al., 2006; Mactier et al., 2007) mentioned above. Machines having performed in excess of $25,000 \mathrm{~h}$ were excluded from this analysis. Areas with an average ATT greater than 30 minutes' drive time were defined as having low accessibility to haemodialysis centres. The reverse summation of patients' ATTs with values $>30$ minutes in each area was expressed as an access

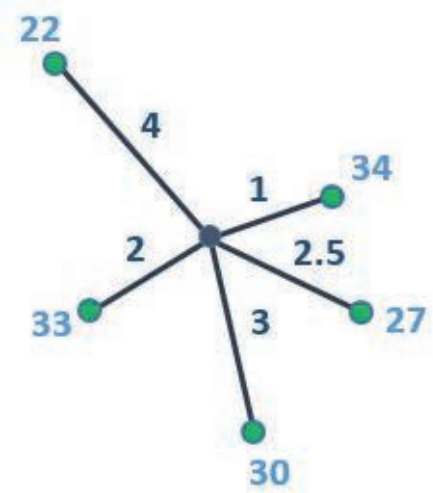

$$
Z(x)=\frac{\sum w_{i} z_{i}}{\sum w_{i}}=\frac{\frac{34}{1^{2}}+\frac{33}{2^{2}}+\frac{27}{2.5^{2}}+\frac{30}{3^{2}}+\frac{22}{4^{2}}}{\frac{1}{1^{2}}+\frac{1}{2^{2}}+\frac{1}{2.5^{2}}+\frac{1}{3^{2}}+\frac{1}{4^{2}}}=32.38
$$

Figure 2. Results of the inverse distance-weighting algorithm for interpolation. 
index ranging from 1 to 9 , where 1 represents low and 9 high access to haemodialysis (Figure 3). This method considered both the number of patients and the ATT of each patient in a region (Eq. 1). It was only applied in areas where with at least one haemodialysis patient.

$$
\text { Access }=\frac{1}{\sum_{i=1}^{n} A T T_{i}}\left(A T T_{i}>30\right)
$$

Eq. 1

\section{Results}

The current availability of haemodialysis in North Khorasan Province is summarised in Table 1, while Table 2 shows the predicted need for new haemodialysis machines at the district level. According this modelled prediction, six new haemodialysis machines are immediately required in North Khorasan Province to provide high-quality services for dialysis patients. The future need is considerably higher, with 5 additional haemodialysis machines next year, followed by 11, 7, 9 and 12 in the following four years, respectively.

The results of executing the IDW algorithm are shown in

Table 1. Current situation with respect to haemodialysis availability in North Khorasan Province, Iran.

\begin{tabular}{lcccccc} 
Area & $\begin{array}{c}\text { Dialysis } \\
\text { centres (n) }\end{array}$ & $\begin{array}{c}\text { Patients } \\
\text { (n) }\end{array}$ & $\begin{array}{c}\text { Available dialysis } \\
\text { machines (n) }\end{array}$ & $\begin{array}{c}\text { Hours used } \\
\text { by each machine* }\end{array}$ & $\begin{array}{c}\text { Machine use } \\
<25,000 \text { hours }\end{array}$ & $\begin{array}{c}\text { Machine use } \\
>25,000 \\
\text { hours }\end{array}$ \\
\hline 1 & 1 & 22 & 5 & 13,827 & 5 & 0 \\
2 & 1 & 17 & 9 & 1776 & 5 & 0 \\
\hline 3 & 3 & 121 & 34 & 15,340 & 31 & 3 \\
4 & 1 & 68 & 12 & 11,876 & 12 & 0 \\
\hline$*$
\end{tabular}

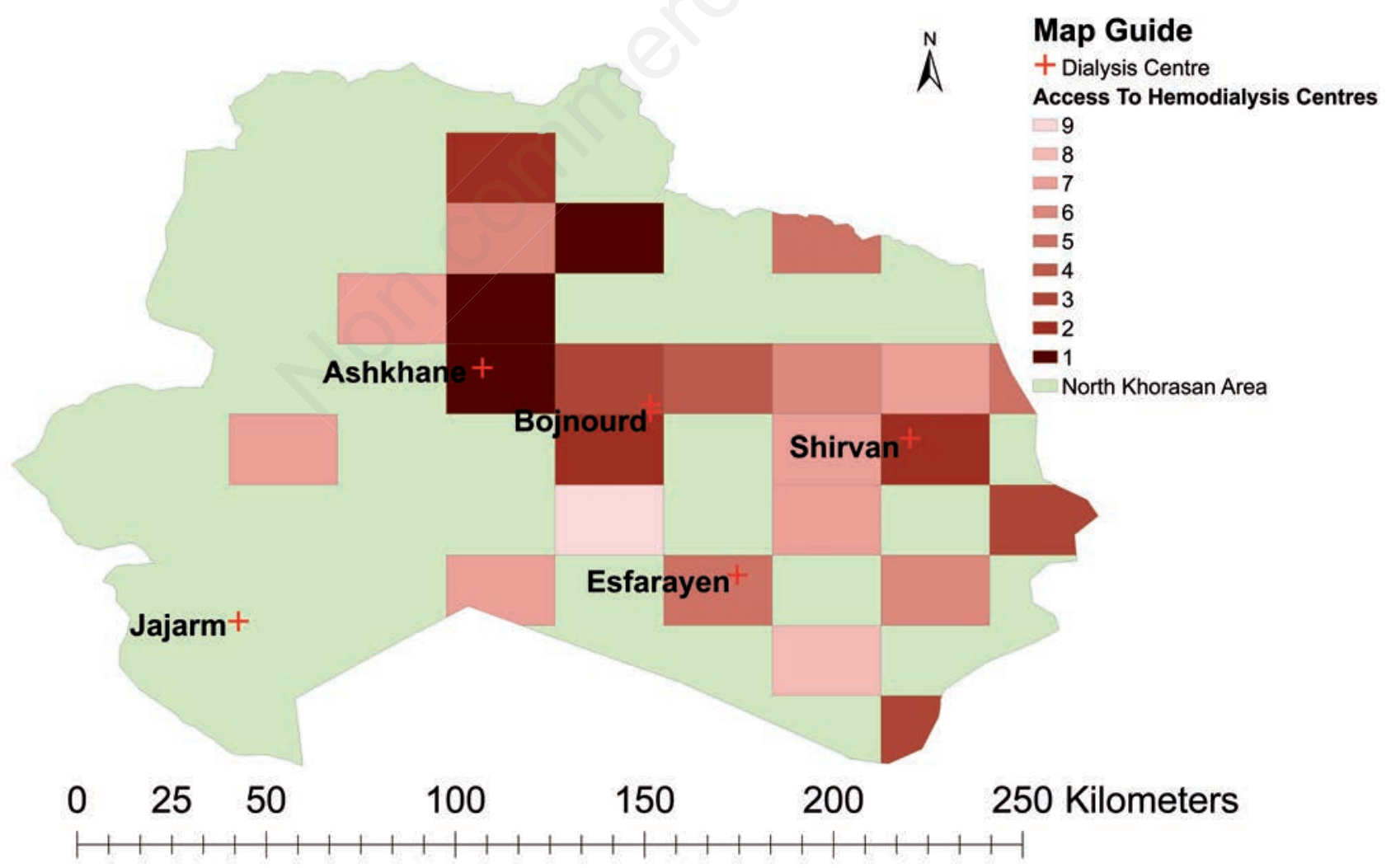

Figure 3. Access to haemodialysis centres in deprived regions of North Khorasan Province, Iran. 
Figure 2. Areas with ATTs less than 30 minutes are shown in green in Figure 4, which highlights suggested haemodialysis machine allocations for these areas. Area 3 had the highest rate of machine usage, whereas Area 2 had the least.

As much as $44 \%$ of patients in North Khorasan have an ATT in excess of 30 minutes, which shows that the ATTs can only be reduced by active intervention. As can be seen in Figure 3, the area around the Ashkhane City was found to have the poorest patient access to haemodialysis.

\section{Discussion}

In this research, the model used self-reported ATT and the actual active hours of haemodialysis machines. This information enables calculation of a reliable estimation of the need for new haemodialysis machines.

We introduced a GIS-based model to visualise the pattern of patient access to haemodialysis and to predict areas in future need of allocation of additional haemodialysis machines. The model classified the study area into two distinct categories: areas with less than 30 minutes access time and those with more. If the resource allocation over the next five years is followed up as recommended by the model, each area's access to the haemodialysis centres will be maintained within present access conditions. Thus, patients' travel time will not increase. Previous studies of haemodialysis access have shown that the number of haemodialysis machines can affect patients' ATTs. For example, Mastsumoto et al. (2012) in Japan considered both distance and facility capacity in their model, while Yang et al. (2006) compared different access measurement methods of patient access in USA. The conclusion is that methods that include the capacity of haemodialysis facilities reflect patients' access better. Therefore, an appropriate allocation of haemodialysis machines in each area is critical to decrease health inequity with respect to haemodialysis service.

Figure 4 shows that a great area proportion (77\%) of North

Table 2. Estimated number of additional haemodialysis machines needed now and in the future in North Khorasan Province, Iran.

\begin{tabular}{lcccccccc} 
Area & Patients needing haemodialysis now & \multicolumn{7}{c}{ Additional haemodialysis machines needed } \\
& & Now & Year 1 & Year 2 & Year 3 & Year 4 & Year 5 \\
1 & 22 & 1 & 0 & 1 & 0 & 1 & 1 \\
2 & 17 & 0 & 0 & 1 & 0 & 1 & 0 \\
\hline 3 & 121 & 0 & 3 & 8 & 5 & 6 & 9 \\
4 & 68 & 5 & 2 & 1 & 2 & 1 & 2 \\
\hline Total & 228 & 6 & 5 & 11 & 7 & 9 & 12 \\
\hline
\end{tabular}

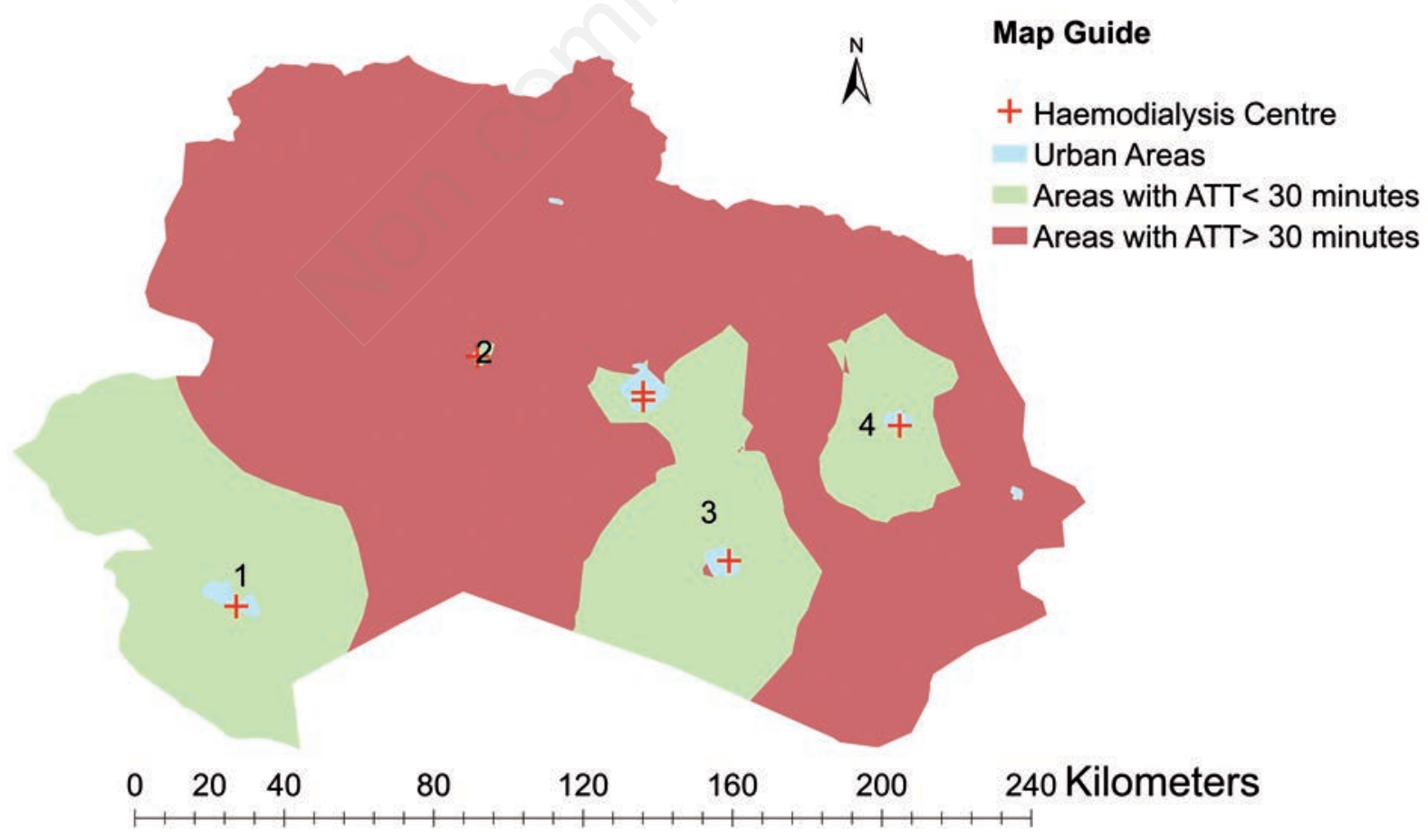

Figure 4. Distribution of areas with respect to actual travel time between patients and haemodialysis centres in North Khorasan Province, Iran. 
Khorasan Province has ATTs of more than 30 minutes. At first glance, this issue can be solved by choosing the best place for establishing new haemodialysis centre for regions experiencing access problems. Our predictive model identifies the area around Ashkhane City as the poorest with regard to haemodialysis and most in need of machine allocation (Figure 3). Of interest in this district is that a new haemodialysis facility there was established only one year ago. However, due to the poor quality of service provision in Ashkhane, patients prefer Bojnord City. Thus, enhancing the quality of haemodialysis service provision in Ashkane would decrease both travel time and cost. We also performed the interpolation of travel time to the closest facility using network analysis instead of ATT. Ashkhane region already owns a haemodialysis centre so this area was not recommended as a poor haemodialysis area due to travel time. We suggest that the calculated travel time to the closest facility is not a good proxy for haemodialysis resource allocation if used alone as there are many other reasons, such as poor transportation (waiting time for vehicles), special-needs patients, e.g., those belonging to lowincome groups, and patients who do not seek the nearest haemodialysis centre.

In this study area, a significant number of patients lived in areas with more than 30 minutes of ATT (Figure 1). Using mobile haemodialysis centres and encouraging/training patients to perform peritoneal dialysis might be a solution to provide this service in a timely manner for these patients. A study by Christie et al. (2005) showed that provision of mobile units in Wales, UK improves the spatial accessibility of renal replacement therapy services. We propose to investigate the cost-efficiency of using mobile haemodialysis for remote areas in order to improve ATTs through alternative efficient solutions.

Although the model in this study is based on information on patients and services within the North Khorasan Province, we believe that our proposed model can be generalised for any area as it estimates the need for additional haemodialysis machines by considering the number of haemodialysis patients and machines in restricted areas. In future studies, it is planned to enhance the capability of the model by defining a haemodialysis need index. For example areas with more patients with diabetes and hypertension conditions are likely to have more CKD and ESRD incidences (Anupama et al., 2017; De Cosmo et al., 2016).

We are aware that future new patients will come from new addresses that cannot be predicted, and therefore the estimated future ATTs is just the best of what one can get at present. In addition, we used the growth rate of haemodialysis patients at the national level (Iran) for the selected area of study. We believe that for a more accurate result, this rate should be adjusted for this province, when local such data become available.

\section{Conclusions}

A GIS-based model can not only be used to investigate the need for new haemodialysis machines, but also to examine geographic disparities in haemodialysis service allocation and to identify areas which are most in need. It is important that policymakers consider both spatial and non-spatial dimensions of access when allocating future haemodialysis services and thus ensure that they target the catchment areas correctly.

\section{References}

Anand S, Khanam MA, Finkelstein FO, 2014. Global perspective of kidney disease. In: L.D. Byham-Gray et al. (eds.) Nutrition in kidney disease. Springer, Berlin, Germany, pp. 11-23.

Anupama Y, Hegde S, Uma G, Patil M, 2017. Hypertension is an important risk determinant for chronic kidney disease: results from a cross-sectional, observational study from a rural population in South India. J Human Hypertens 31:327-32

Ayyalasomayajula B, Wiebe N, Hemmelgarn BR, Bello A, Manns B, Klarenbach S, Tonelli M, 2011. A novel technique to optimize facility locations of new nephrology services for remote areas. Clin J Am Soc Nephrol 6:2157-64.

Azar AT, 2009. The influence of maintenance quality of hemodialysis machines on hemodialysis efficiency. Saudi J Kidney Dis Transpl 20:49.

Christie S, Morgan G, Heaven M, Sandifer Q, van Woerden H, 2005. Analysis of renal service provision in south and mid Wales. Publ Health 119:738-42.

Daugirdas JT, Depner TA, Inrig J, Mehrotra R, Rocco MV, Suri RS, Weiner DE, Greer N, Ishani A, MacDonald R, Olson C, Rutks I, Slinin Y, Wilt TJ, Rocco M, Kramer H, Choi MJ, Samaniego-Picota M, Scheel PJ, Willis K, Joseph J, Brereton L, 2015. KDOQI clinical practice guideline for hemodialysis adequacy: 2015 update. Am J Kidney Dis 66:884-930.

De Cosmo S, Viazzi F, Pacilli A, Giorda C, Ceriello A, Gentile S, Russo G, Rossi MC, Nicolucci A, Guida P, 2016. Predictors of chronic kidney disease in type 2 diabetes: a longitudinal study from the AMD Annals initiative. Medicine 95:e4007.

Delmelle EM, Cassell CH, Dony C, Radcliff E, Tanner JP, Siffel C, Kirby RS, 2013. Modeling travel impedance to medical care for children with birth defects using Geographic Information Systems. Birth Def Res A 97:673-84.

Diamant MJ, Harwood L, Movva S, Wilson B, Stitt L, Lindsay RM, Moist LM, 2010. A comparison of quality of life and travel-related factors between in-center and satellite-based hemodialysis patients. Clin J Am Soc Nephrol 5:268-74.

Hill NR, Fatoba ST, Oke JL, Hirst JA, O'Callaghan CA, Lasserson DS, Hobbs FR, 2016. Global prevalence of chronic kidney disease: a systematic review and meta-analysis. PLoS One 11:e0158765.

Jia P, Anderson JD, Leitner M, Rheingans R, 2016. High-resolution spatial distribution and estimation of access to improved sanitation in Kenya. PloS One 11:e0158490.

Mactier R, Hoenich N, Breen C, 2007. Clinical practice guidelines: 3a - haemodialysis. UK Renal Association Clinical Practice Guidelines. UK Renal Association, Bristol, UK.

Maheswaran R, Payne N, Meechan D, Burden R, Fryers P, Wight J, Hutchinson A, 2003. Socioeconomic deprivation, travel distance, and renal replacement therapy in the Trent Region, United Kingdom 2000: an ecological study. J Epidemiol Commun Health 57:523-4.

Mao L, Nekorchuk D, 2013. Measuring spatial accessibility to healthcare for populations with multiple transportation modes. Health Place 24:115-22.

Matsumoto M, Kashima S, Ogawa T, Takeuchi K, 2013. Do rural and remote areas really have limited accessibility to health care? Geographic analysis of dialysis patients in Hiroshima, Japan. Rural Remote Health 13:2507.

Matsumoto M, Ogawa T, Kashima S, Takeuchi K, 2012. The impact of rural hospital closures on equity of commuting time 
for haemodialysis patients: simulation analysis using the capacity-distance model. Int J Health Geogr 11:28.

Moist LM, Bragg-Gresham JL, Pisoni RL, Saran R, Akiba T, Jacobson SH, Fukuhara S, Mapes DL, Rayner HC, Saito A, 2008. Travel time to dialysis as a predictor of health-related quality of life, adherence, and mortality: the Dialysis Outcomes and Practice Patterns Study (DOPPS). Am J Kidney Dis 51:641-50.

Omrani-Khoo H, Lotfi F, Safari H, Jame SZB, Moghri J, Shafii M, 2013. Equity in distribution of health care resources; assessment of need and access, using three practical indicators. Iran J Public Health 42:1299.

Rucker D, Hemmelgarn BR, Lin M, Manns BJ, Klarenbach SW, Ayyalasomayajula B, James MT, Bello A, Gordon D, Jindal KK, 2011. Quality of care and mortality are worse in chronic kidney disease patients living in remote areas. Kidney Int 79:210-7.

Saunders MR, Lee H, Maene C, Schuble T, Cagney KA, 2014. Proximity does not equal access: racial disparities in access to high quality dialysis facilities. J Racial Ethnic Health Disp
$1: 291-9$

Stentzel U, Piegsa J, Fredrich D, Hoffmann W, Berg N, 2016. Accessibility of general practitioners and selected specialist physicians by car and by public transport in a rural region of Germany. BMC Health Serv Res 16:587.

Stephens JM, Brotherton S, Dunning SC, Emerson LC, Gilbertson DT, Harrison DJ, Kochevar JJ, McClellan AC, McClellan WM, Wan S, Gitlin M, 2013. Geographic disparities in patient travel for dialysis in the United States. J Rural Health 29:33948.

Thompson S, Gill J, Wang X, Padwal R, Pelletier R, Bello A, Klarenbach S, Tonelli M, 2012. Higher mortality among remote compared to rural or urban dwelling hemodialysis patients in the United States. Kidney Int 82:352-9.

Wikipedia, 2016. North Khorasan Province. Available from: https://en.wikipedia.org/wiki/North_Khorasan_Province

Yang D-H, Goerge R, Mullner R, 2006. Comparing GIS-based methods of measuring spatial accessibility to health services. $\mathrm{J}$ Med Syst 30:23-32. 